\title{
Glycerol or crude glycerol as substrates make Pseudomonas aeruginosa achieve anaerobic production of rhamnolipids
}

\author{
Feng Zhao ${ }^{*} \mathbb{D}$, Yuting Wu', Qingzhi Wang ${ }^{1}$, Mengyao Zheng ${ }^{1}$ and Qingfeng Cui ${ }^{2}$
}

\begin{abstract}
Background: The anaerobic production of rhamnolipids is significant in research and application, such as foamless fermentation and in situ production of rhamnolipids in the anoxic environments. Although a few studies reported that some rare Pseudomonas aeruginosa strains can produce rhamnolipids anaerobically, the decisive factors for anaerobic production of rhamnolipids were unknown.
\end{abstract}

Results: Two possible hypotheses on the decisive factors for anaerobic production of rhamnolipids by $P$. aeruginosa were proposed, the strains specificity of rare P. aeruginosa (hypothesis 1 ) and the effect of specific substrates (hypothesis 2). This study assessed the anaerobic growth and rhamnolipids synthesis of three $P$. aeruginosa strains using different substrates. P. aeruginosa strains anaerobically grew well using all the tested substrates, but glycerol was the only carbon source that supported anaerobic production of rhamnolipids. Other carbon sources with different concentrations still failed for anaerobic production of rhamnolipids by $P$. aeruginosa. Nitrate was the excellent nitrogen source for anaerobic production of rhamnolipids. FTIR spectra analysis confirmed the anaerobically produced rhamnolipids by $P$. aeruginosa using glycerol. The anaerobically produced rhamnolipids decreased air-water surface tension to below $29.0 \mathrm{mN} / \mathrm{m}$ and emulsified crude oil with $\mathrm{El}_{24}$ above 65\%. Crude glycerol and 1, 2-propylene glycol also supported the anaerobic production of rhamnolipids by all P. aeruginosa strains. Prospects and bottlenecks to anaerobic production of rhamnolipids were also discussed.

Conclusions: Glycerol substrate was the decisive factor for anaerobic production of rhamnolipids by P. aeruginosa. Strain specificity resulted in the different anaerobic yield of rhamnolipids. Crude glycerol was one low cost substrate for anaerobic biosynthesis of rhamnolipids by P. aeruginosa. Results help advance the research on anaerobic production of rhamnolipids, deepen the biosynthesis theory of rhamnolipids and optimize the anaerobic production of rhamnolipids.

Keywords: Rhamnolipids, Pseudomonas aeruginosa, Anaerobic production, Glycerol, Strain specificity

\section{Background}

Rhamnolipids is the most extensively studied biosurfactants nowadays [1-3]. Due to its relatively high yield and good activity, rhamnolipids has great application

*Correspondence: zhaofeng2019@qfnu.edu.cn

1 School of Life Sciences, Qufu Normal University, Qufu 273165, Shandong, China

Full list of author information is available at the end of the article potential in enhanced oil recovery, bioremediation, agriculture, food, cosmetic, and other fields [3-6]. The anaerobic production of rhamnolipids is significant in research and application, such as in situ production of rhamnolipids in the anoxic environments and foamless fermentation.

Oil reservoirs, deep soil and sediments are anoxic environments. The anaerobic production of rhamnolipids can achieve in situ production of rhamnolipids in such original author(s) and the source, provide a link to the Creative Commons licence, and indicate if changes were made. The images or other third party material in this article are included in the article's Creative Commons licence, unless indicated otherwise in a credit line to the material. If material is not included in the article's Creative Commons licence and your intended use is not permitted by statutory regulation or exceeds the permitted use, you will need to obtain permission directly from the copyright holder. To view a copy of this licence, visit http://creativecommons.org/licenses/by/4.0/. The Creative Commons Public Domain Dedication waiver (http://creativeco mmons.org/publicdomain/zero/1.0/) applies to the data made available in this article, unless otherwise stated in a credit line to the data. 
environments [7-9]. Rhamnolipids is a good oil displacement agent for enhanced oil recovery due to its excellent emulsifying activity to crude oil and surface activity to reducing interfacial tension of water/oil/rock [10]. In situ production of rhamnolipids in oil reservoirs is more costeffective and easy to operate for enhanced oil recovery [11-13]. Producing rhamnolipids anaerobically by microorganisms can achieve in situ production of rhamnolipids in anoxic oil reservoirs.

The foam problem in aerobic fermentation has been perplexing the rhamnolipids production [14]. During the aerobic production of rhamnolipids, foam is minute bubbles mainly formed by rhamnolipids liquid membrane filling air. Adding defoamer can just solve the foam problem at some extent $[14,15]$. Moreover, rhamnolipids fermentation without aeration can avoid the foam formation and develop foamless fermentation. Anaerobic production of rhamnolipids can achieve the foamless fermentation of rhamnolipids [7].

Rhamnolipids-producing bacteria mainly including Burkholderia sp. and Pseudomonas sp. [1, 3]. At present, Pseudomonas aeruginosa is considered to be the most productive rhamnolipids producer [1,3], which makes $P$. aeruginosa become the research focus. $P$. aeruginosa is one of facultative anaerobic bacteria [7, 16, 17]. Although $P$. aeruginosa can grow at both aerobic and anaerobic conditions, studies on rhamnolipids production by $P$. aeruginosa were focused on the aerobic conditions [7]. Only a few studies reported that some rare $P$. aeruginosa strains can produce rhamnolipids anaerobically, such as P. aeruginosa ATCC10145, Pseudomonas sp ANBIOSURF-1, $P$. aeruginosa SG $[8,9,13,18-21]$.

Can other $P$. aeruginosa strains produce rhamnolipids anaerobically? The decisive factors for anaerobic production of rhamnolipids were still unknown. Revealing the decisive factors are helpful to advance the research on anaerobic production of rhamnolipids.

In this study, two possible hypotheses on the decisive factors for anaerobic production of rhamnolipids were proposed, the strains specificity of $P$. aeruginosa (hypothesis 1 ) and the effect of specific substrates (hypothesis 2). Using diverse substrates, anaerobic growth and rhamnolipids production of different $P$. aeruginosa strains were investigated. Three $P$. aeruginosa strains isolated from different sources were used. Diverse carbon sources and nitrogen sources were tested for anaerobic production of rhamnolipids. Anaerobically produced rhamnolipids was confirmed by Fourier transform infrared (FTIR) spectra analysis. The physicochemical properties of rhamnolipids products were also evaluated. Prospects and bottlenecks of anaerobic production of rhamnolipids were also discussed. Results would deepen the biosynthesis theory of rhamnolipids and guide the optimization process for anaerobic production of rhamnolipids.

\section{Materials and methods}

Strains and culture conditions

In this study, three P. aeruginosa strains (SG, L6-1 and FA1) isolated from different sources were investigated for rhamnolipids production. Strain P. aeruginosa SG and L6-1 were respectively isolated from production fluid of different oil reservoirs in Xinjiang Oilfield, China [8]. Strains $P$. aeruginosa FA1 were isolated from agricultural soil in Shandong province, China. The phylogenetic relationship of the three $P$. aeruginosa strains was shown in Fig. 1. P. aeruginosa SG was used as the positive control for anaerobic production of rhamnolipids using glycerol [8]. LB medium was used to prepare the seed culture at $35{ }^{\circ} \mathrm{C}$ and $180 \mathrm{rpm}$. For anaerobic cultivation, $P$. aeruginosa strains were cultured in $100 \mathrm{ml}$ serum bottles containing $80 \mathrm{ml}$ anaerobic medium. The inoculum amount was $3 \%$. Briefly, the anaerobic medium was prepared as follows [8]. The medium was boiled for $15 \mathrm{~min}$, and then 99.99\% purity of $\mathrm{N}_{2}$ gas was injected into the boiling medium for 5 min to drive out oxygen. Then medium was sub-packaged in serum bottles when it was hot. Press the rubber plug and press the aluminum cap under $\mathrm{N}_{2}$ gas protection. After sterilization and cooling to $30{ }^{\circ} \mathrm{C}$,

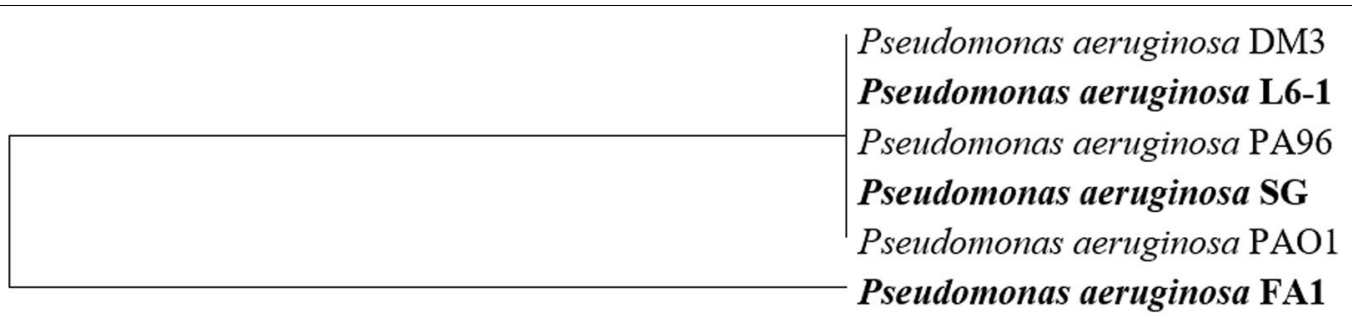

$\stackrel{\longmapsto}{ }$

Fig. 1 Neighbour-joining phylogenetic tree of three P. aeruginosa strains constructed by software Mega 5.0 based on a neighbour-joining analysis of 1000 resampled datasets. Bar, 0.00005 nucleotide substitutions per site 
deoxidizer $\mathrm{Na}_{2} \mathrm{~S} \cdot 9 \mathrm{H}_{2} \mathrm{O}$ was added into the medium to a final concentration of $0.02 \%(\mathrm{w} / \mathrm{v})$ [20]. The anaerobic fermentation experiments were performed at $35{ }^{\circ} \mathrm{C}$ and $50 \mathrm{rpm}$ for 10 days. Three parallel experiments were set for each bacterial strain. The non-inoculated medium was used as the negative control.

\section{Analytical methods}

Anaerobic culture samples were taken from serum bottles using sterile syringes. Bacterial biomass was represented using $\mathrm{OD}_{600}$ values of the anaerobic culture. Culture samples were centrifuged at $10,000 \mathrm{~g}$ and $10{ }^{\circ} \mathrm{C}$ for $10 \mathrm{~min}$. The surface tension of supernatant was measured by surface tensiometer (BZY-1, Shanghai Hengping Instrument and Meter Factory, Shanghai, China). The diameter of oil spreading circle formed by culture supernatant was determined as previous reported [22]. The used crude oil (7-Middle area, Xinjiang oilfield, China) has the density of $0.823 \mathrm{~g} / \mathrm{ml}$ and the viscosity of 8.6 $\mathrm{mPa} \cdot \mathrm{s}$ at $30{ }^{\circ} \mathrm{C}$. Emulsification index $\left(\mathrm{EI}_{24}\right)$ was measured to evaluate the emulsifying activity of anaerobically produced rhamnolipids [20]. $\mathrm{EI}_{24}$ values were calculated as the height of the emulsified layer $(\mathrm{mm})$ divided by the total height of the liquid column $(\mathrm{mm})$ and multiplied by 100.

\section{P. aeruginosa strains fed with different carbon sources}

In this study, the commonly used carbon sources for aerobic production of rhamnolipids were investigated for anaerobic production of rhamnolipids. The selected carbon sources were glucose, glycerol, palmitic acid and soybean oil. The concentrations of carbon sources in the medium were all $40 \mathrm{~g} / \mathrm{l}$. The medium except carbon source contained $4 \mathrm{~g} / \mathrm{l}$ of $\mathrm{NaNO}_{3}, 4 \mathrm{~g} / \mathrm{l}$ of $\mathrm{K}_{2} \mathrm{HPO}_{4} \cdot 3 \mathrm{H}_{2} \mathrm{O}$, $3 \mathrm{~g} / \mathrm{l}$ of $\mathrm{KH}_{2} \mathrm{PO}_{4}, 0.5 \mathrm{~g} / \mathrm{l}$ of $\mathrm{MgSO}_{4} \cdot 7 \mathrm{H}_{2} \mathrm{O}, 0.5 \mathrm{~g} / \mathrm{l}$ of $\mathrm{KCl}$, $0.5 \mathrm{~g} / \mathrm{l}$ of NaCl , and $0.2 \mathrm{~g} / \mathrm{l}$ of $\mathrm{CaCl}_{2} \cdot 2 \mathrm{H}_{2} \mathrm{O}$. After anaerobic cultivation, the $\mathrm{OD}_{600}$, surface tension and oil spreading activity were analyzed. Anaerobic production of rhamnolipids was defined as reducing the surface tension of anaerobic culture to lower than $35 \mathrm{mN} / \mathrm{m}$ and forming oil spreading circles with diameters greater than $15 \mathrm{~mm}$.

\section{P. aeruginosa strains cultured using carbon sources with different concentrations}

For the tested carbon sources f, using a single concentration maybe not persuasive. Glucose, glycerol, palmitic acid and soybean oil with concentrations of $15 \mathrm{~g} / \mathrm{l}$ and $60 \mathrm{~g} / \mathrm{l}$ were also studied for anaerobic production of rhamnolipids by three $P$. aeruginosa strains. The medium except carbon source contained $4 \mathrm{~g} / \mathrm{l}$ of $\mathrm{NaNO}_{3}, 4 \mathrm{~g} / \mathrm{l}$ of $\mathrm{K}_{2} \mathrm{HPO}_{4} \cdot 3 \mathrm{H}_{2} \mathrm{O}, 3 \mathrm{~g} / \mathrm{l}$ of $\mathrm{KH}_{2} \mathrm{PO}_{4}, 0.5 \mathrm{~g} / \mathrm{l}$ of $\mathrm{MgSO}_{4} \cdot 7 \mathrm{H}_{2} \mathrm{O}$, $0.5 \mathrm{~g} / \mathrm{l}$ of KCl, $0.5 \mathrm{~g} / \mathrm{l}$ of NaCl , and $0.2 \mathrm{~g} / \mathrm{l}$ of $\mathrm{CaCl}_{2} \cdot 2 \mathrm{H}_{2} \mathrm{O}$.
After anaerobic cultivation, the surface tension and oil spreading activity of culture samples were analyzed.

\section{P. aeruginosa strains fed with different nitrogen sources}

The common nitrogen nutrients, $\mathrm{NH}_{4} \mathrm{Cl}, \mathrm{NaNO}_{3}$, yeast extract and peptone, were tested for the anaerobic production of rhamnolipids by $P$. aeruginosa strains. Strains $P$. aeruginosa SG was used as the positive control. The concentrations of nitrogen sources in the medium were all $4 \mathrm{~g} / \mathrm{l}$. The complex nitrogen sources of $\mathrm{NaNO}_{3}$ and yeast extract $(3: 7,5: 5,7: 3)$ were also tested for anaerobic production of rhamnolipids by $P$. aeruginos a strains. The amount of complex nitrogen sources was also $4 \mathrm{~g} / \mathrm{l}$. The medium except nitrogen source contained $40 \mathrm{~g} / \mathrm{l}$ of glycerol, $4 \mathrm{~g} / \mathrm{l}$ of $\mathrm{K}_{2} \mathrm{HPO}_{4} \cdot 3 \mathrm{H}_{2} \mathrm{O}, 3 \mathrm{~g} / \mathrm{l}$ of $\mathrm{KH}_{2} \mathrm{PO}_{4}, 0.5 \mathrm{~g} / \mathrm{l}$ of $\mathrm{MgSO}_{4} \cdot 7 \mathrm{H}_{2} \mathrm{O}, 0.5 \mathrm{~g} / \mathrm{l}$ of $\mathrm{KCl}, 0.5 \mathrm{~g} / \mathrm{l}$ of $\mathrm{NaCl}$, and $0.2 \mathrm{~g} / \mathrm{l}$ of $\mathrm{CaCl}_{2} \cdot 2 \mathrm{H}_{2} \mathrm{O}$. The $\mathrm{OD}_{600}$ values, surface tension and oil spreading activity of culture samples were analyzed after anaerobic cultivation.

\section{Rhamnolipids extraction from the anaerobic culture}

The anaerobically produced rhamnolipids were extracted as previous references described $[9,23]$. Briefly, the cell free anaerobic culture broth was heated at $80{ }^{\circ} \mathrm{C}$ for $30 \mathrm{~min}$ to denature the extracellular proteins. Samples were centrifuged at 10,000 $\mathrm{g}$ for $10 \mathrm{~min}$. Using $6 \mathrm{~mol} / \mathrm{l}$ of $\mathrm{HCl}$-water solution, the $\mathrm{pH}$ value of samples was adjusted to 2.0. The organic solvent, chloroform and methanol (v/v, 2:1), was used for rhamnolipids extraction from aqueous phase. The rhamnolipids products were recovered from the organic phase using a rotary evaporator $\left(50 \mathrm{rpm}, 45^{\circ} \mathrm{C}\right)$.

\section{FTIR spectra analysis}

Anaerobically produced rhamnolipids was confirmed by FTIR spectra analysis. Anaerobically produced rhamnolipids from $P$. aeruginosa SG was used as the positive control. Briefly, $10 \mathrm{mg}$ of rhamnolipids extract and $90 \mathrm{mg}$ of $\mathrm{KBr}$ was mixed to make the translucent pellet with pressure of $25 \mathrm{Mpa}$ for $25 \mathrm{~s}$. A NICOLET 380 FTIR spectrometer was used to record the FTIR spectra of the translucent pellet with the wave number from $400 \mathrm{~cm}^{-1}$ to $4000 \mathrm{~cm}^{-1}[9,20]$.

\section{Feasibility of glycerol intermediates and crude glycerol for anaerobic production of rhamnolipids}

The possible glycerol intermediates, hydroxyacetone, 1, 2-propylene glycol and 1, 3-propylene glycol, were evaluated as carbon sources for anaerobic production of rhamnolipids by three $P$. aeruginosa strains. The concentration of glycerol intermediates was $40 \mathrm{~g} / \mathrm{l}$. The medium except carbon source contained $4 \mathrm{~g} / \mathrm{l}$ of $\mathrm{NaNO}_{3}, 4 \mathrm{~g} / \mathrm{l}$ of $\mathrm{K}_{2} \mathrm{HPO}_{4} \cdot 3 \mathrm{H}_{2} \mathrm{O}, 3 \mathrm{~g} / \mathrm{l}$ of $\mathrm{KH}_{2} \mathrm{PO}_{4}, 0.5 \mathrm{~g} / \mathrm{l}$ of $\mathrm{MgSO}_{4} \cdot 7 \mathrm{H}_{2} \mathrm{O}$, 
$0.5 \mathrm{~g} / \mathrm{l}$ of KCl, $0.5 \mathrm{~g} / \mathrm{l}$ of NaCl, $0.2 \mathrm{~g} / \mathrm{l}$ of and $\mathrm{CaCl}_{2} \cdot 2 \mathrm{H}_{2} \mathrm{O}$. Crude glycerol was also attempted for anaerobic production of rhamnolipids by $P$. aeruginosa strains. The tested crude glycerol containing $95 \%$ of glycerol. The concentration of crude glycerol in the medium was $40 \mathrm{~g} / \mathrm{l}$. After anaerobic cultivation, the $\mathrm{OD}_{600}$, surface tension and oil spreading activity of culture samples were analyzed.

\section{Results and discussion}

\section{Anaerobic production of rhamnolipids using different} carbon sources

Using the tested carbon sources, the biomass $\left(\mathrm{OD}_{600}\right)$, surface activity and oil spreading activity were shown in Fig. 2. Using different carbon sources, three $P$. aeruginosa strains obtained biomass with $\mathrm{OD}_{600}$ values between 1.80 and 4.00 under anaerobic conditions (Fig. 2a). Results indicated that three $P$. aeruginosa strains can anaerobically grow well using all the tested carbon sources. Results also confirmed that $P$. aeruginosa are facultative anaerobic bacteria $[7,16,17]$. Among the tested carbon

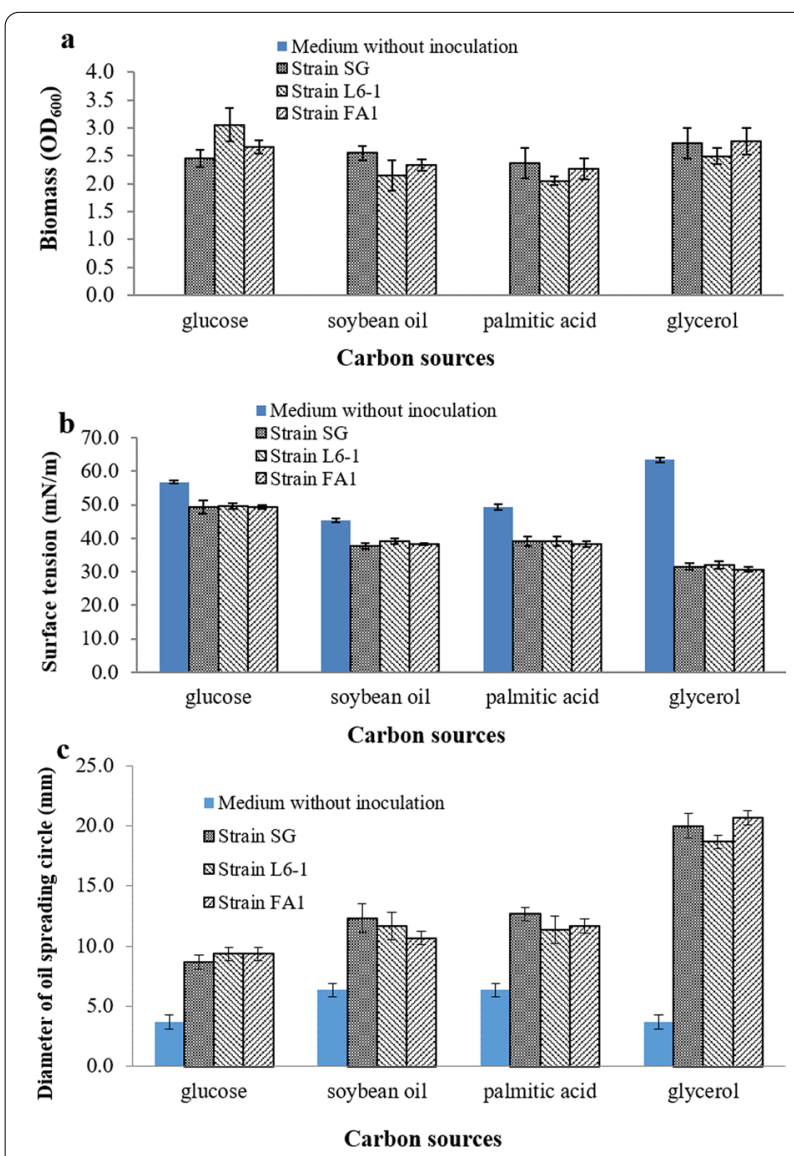

Fig. 2 Anaerobic growth and production of rhamnolipids by $P$. aeruginosa strains using different carbon sources: a biomass $\left(\mathrm{OD}_{600}\right)$, b surface activity and c oil spreading activity. Strain P. aeruginosa SG was used as the positive control sources, glucose and glycerol were more favorable to the anaerobic growth of $P$. aeruginosa. As water-soluble substrates, glucose and glycerol are more conducive to be rapid absorbed and metabolized. As shown in Fig. 2b, the surface tension of anaerobic culture was decreased to lower than $35 \mathrm{mN} / \mathrm{m}$ only using glycerol (from $63 \mathrm{mN} / \mathrm{m}$ to about $32 \mathrm{mN} / \mathrm{m}$ ). Using glycerol, $P$. aeruginosa strains reduced the surface tension of anaerobic culture with the greatest decrease (49.5\%). And the surface tension values were lowest. As shown in Fig. 2c, the diameters of oil spreading circles formed by anaerobic culture of three strains were greater than $15 \mathrm{~mm}$ when using glycerol as carbon source. Using other carbon sources, the forming oil spreading circles with diameters smaller than $10 \mathrm{~mm}$. The formed oil spreading circles diameters can be used to indirectly determine biosurfactants concentration in bacterial culture [22]. Using glycerol, the oil spreading circles diameters formed by three $P$. aeruginosa strains, SG, L6-1 and FA1, were $20 \mathrm{~mm}, 18 \mathrm{~mm}$ and $21 \mathrm{~mm}$, respectively (Fig. 2c). Three P. aeruginosa strains, SG, L6-1 and FA1, produced $228 \mathrm{mg} / \mathrm{l}, 195 \mathrm{mg} / \mathrm{l}$ and $244 \mathrm{mg} / \mathrm{l}$ of rhamnolipids using glycerol under anaerobic conditions. Results were compared with single factor analysis of variance using software SPSS. Regarding to all three $P$. aeruginosa strains, the effect of carbon sources on anaerobic production of rhamnolipids was significant $(P<0.01)$. Using glucose, palmitic acid or soybean oil as carbon sources, the effect of strains on anaerobic production of rhamnolipids was not significant $(P>0.1)$. But the effect of strains on anaerobic production of rhamnolipids was significant $(P<0.05)$ when using glycerol as carbon sources. Results demonstrated that $P$. aeruginosa strains can produce rhamnolipids anaerobically when using glycerol as carbon source. Strain specificity resulted in the different anaerobic yield of rhamnolipids when using glycerol as carbon source.

Although all the tested substrates can be used for rhamnolipids production by $P$. aeruginosa under aerobic conditions [23-25], only glycerol can be used for anaerobic production of rhamnolipids by $P$. aeruginosa. Previous studies reported that strain P. aeruginosa SG can anaerobically produce rhamnolipids using glycerol $[8,21]$. In this study, except for strain SG, other P. aeruginosa strains, L6-1 and FA1, can also anaerobically produce rhamnolipids using glycerol. Results confirmed that hypothesis 2 is credible. The specific substrate, glycerol, make $P$. aeruginosa strains achieve the anaerobic production of rhamnolipids.

\section{The effect of carbon sources concentrations on anaerobic production of rhamnolipids}

As shown in Fig. 3a, using glycerol with both concentrations of $15 \mathrm{~g} / \mathrm{l}$ and $60 \mathrm{~g} / \mathrm{l}$, all three $P$. aeruginosa strains 


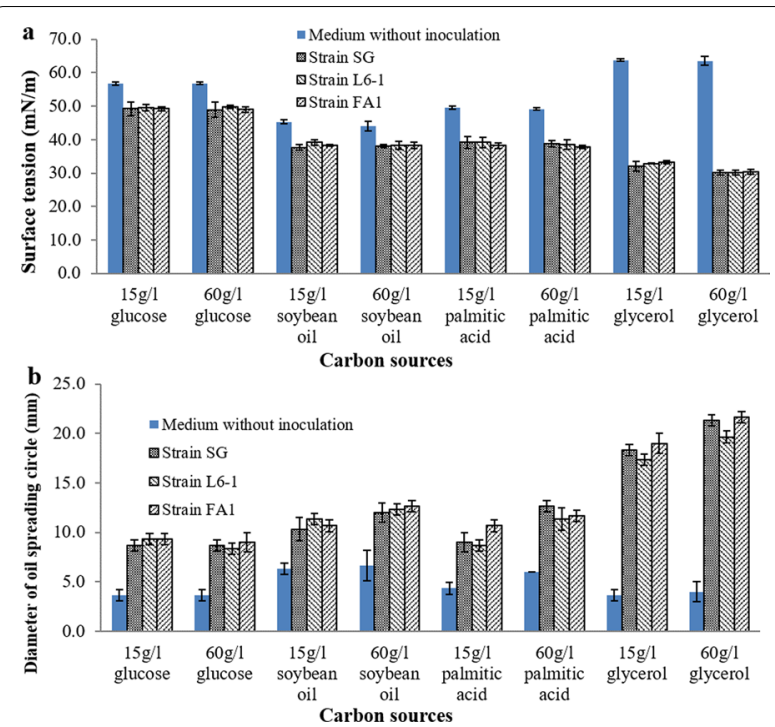

Fig. 3 Effect of different carbon sources concentrations on anaerobic production of rhamnolipids by $P$. aeruginosa strains: a surface activity and $\mathbf{b}$ oil spreading activity reduced the surface tension of anaerobic culture with the greatest decrease, from $63 \mathrm{mN} / \mathrm{m}$ to lower than 32 $\mathrm{mN} / \mathrm{m}$. However, using other carbon sources at both concentrations of $15 \mathrm{~g} / \mathrm{l}$ and $60 \mathrm{~g} / \mathrm{l}$, the surface tension of anaerobic culture was decreased slightly. As shown in Fig. 3b, the diameters of oil spreading circles formed by three strains were greater than $15 \mathrm{~mm}$ when using $15 \mathrm{~g} / \mathrm{l}$ and $60 \mathrm{~g} / \mathrm{l}$ of glycerol. Using other carbon sources at both concentrations of $15 \mathrm{~g} / \mathrm{l}$ and $60 \mathrm{~g} / \mathrm{l}$, the forming oil spreading circles with diameters smaller than $10 \mathrm{~mm}$. Results once again confirmed that three $P$. aeruginosa strains can anaerobically produce rhamnolipids using glycerol as carbon source. Although other carbon sources with different concentrations were used, the tested carbon sources can not support anaerobic production of rhamnolipids by three $P$. aeruginosa strains yet.

Results of the present study demonstrated that glycerol was the only carbon source that supported anaerobic production of rhamnolipids by $P$. aeruginosa. The hypothesis 2 is correct. Glycerol substrate rather than the strain specificity contributed to the anaerobic production of rhamnolipids by $P$. aeruginosa.

\section{Anaerobic production of rhamnolipids using different nitrogen sources}

Using the tested nitrogen sources, the biomass $\left(\mathrm{OD}_{600}\right)$, surface activity and oil spreading activity were shown in Fig. 4. Under anaerobic conditions and using glycerol as carbon source, three $P$. aeruginosa strains obtained biomass with $\mathrm{OD}_{600}$ values between 2.50 and 4.50 using
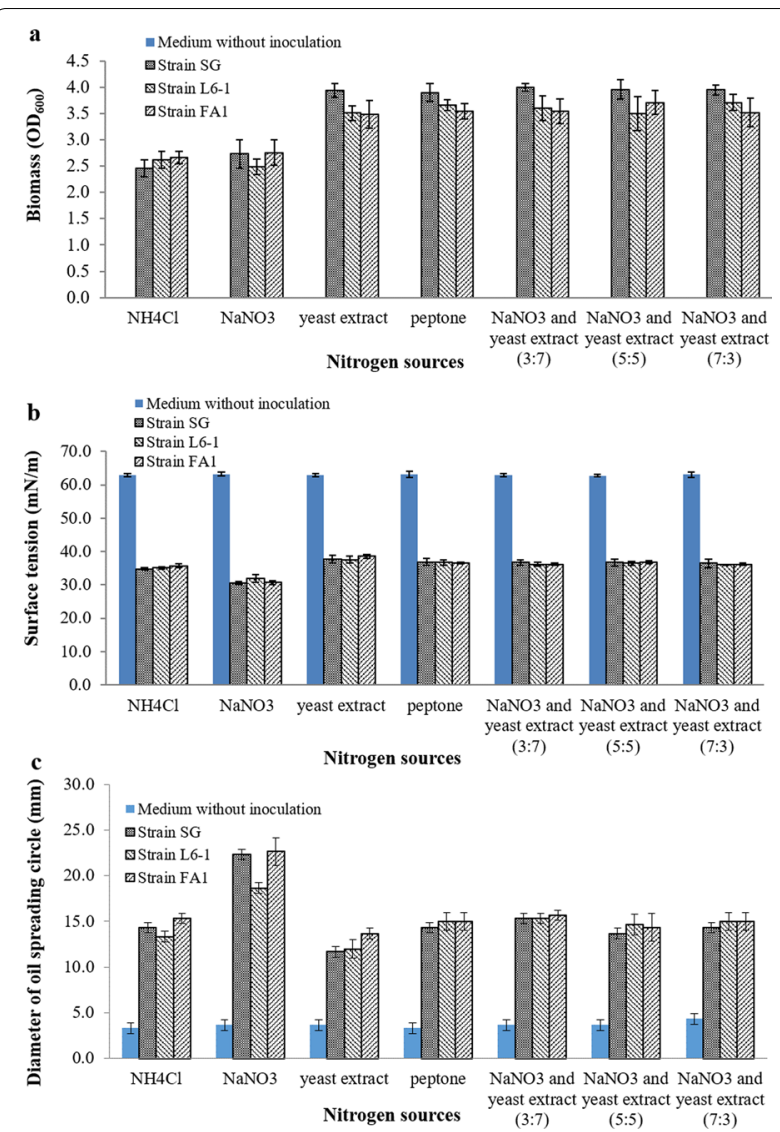

Fig. 4 Anaerobic growth and production of rhamnolipids by $P$. aeruginosa strains using different nitrogen sources: a biomass $\left(\mathrm{OD}_{600}\right)$, b surface activity and coil spreading activity

different nitrogen sources (Fig. 4a). Three $P$. aeruginosa strains anaerobically grew well using different nitrogen sources. As shown in Fig. 4a, the organic nitrogen sources were more favorable to the anaerobic growth of $P$. aeruginosa strains. The small molecular peptides contained in yeast extract and peptone may provide abundant and available nutrition for cell growth of $P$. aeruginosa [26]. As shown in Fig. 4b, the surface tension of anaerobic culture was reduced from $63 \mathrm{mN} / \mathrm{m}$ to lower than 40 $\mathrm{mN} / \mathrm{m}$ using all tested nitrogen sources. Using glycerol as carbon source, $P$. aeruginosa strains anaerobically produced rhamnolipids using the tested nitrogen sources. Results also showed that glycerol is the key factor for anaerobic production of rhamnolipids by $P$. aeruginosa. Using $\mathrm{NaNO}_{3}$ as nitrogen source, $P$. aeruginosa strains reduced the surface tension of anaerobic culture to the lowest surface tension $(31 \mathrm{mN} / \mathrm{m})$, with the greatest decrease (51.3\%). The oil spreading activity were shown in Fig. 4c. Using $\mathrm{NaNO}_{3}$ as nitrogen source, anaerobic culture of $P$. aeruginosa strains formed oil spreading circles with diameters about $20 \mathrm{~mm}$. Results demonstrated 
that nitrate was a favorable nitrogen source for anaerobic production of rhamnolipids by $P$. aeruginosa when using glycerol as carbon source.

Although $P$. aeruginosa strains obtained higher biomass using the complex nitrogen sources of $\mathrm{NaNO}_{3}$ and yeast extract (Fig. 4a), the anaerobic production of rhamnolipids were relatively less (Fig. 4c). The organic nitrogen source may provide abundant and available nutrition for cell growth [26]. But studies also reported that organic nitrogen sources were not conducive to synthesize rhamnolipids [27]. Rhamnolipids is one secondary metabolite. Nitrate was reported to be the best nitrogen source for aerobic production of rhamnolipids $[28,29]$. In this study, nitrate was also the excellent nitrogen source for anaerobic production of rhamnolipid. $P$. aeruginosa can grow and metabolize at both aerobic and anaerobic conditions $[16,17]$. Because $P$. aeruginosa can use other electron acceptors except oxygen, such as nitrate $[7,18]$. Nitrate was one good electron acceptors for anaerobic metabolism of $P$. aeruginosa.

\section{FTIR spectra analysis of anaerobically produced rhamnolipids}

The anaerobically produced rhamnolipids from $P$. aeruginosa strains using glycerol were confirmed by FT-IR spectra analysis, respectively. Rhamnolipids produced by $P$. aeruginosa SG was used as the positive control. As shown in Fig. 5, the FTIR spectra of the anaerobically produced rhamnolipids from $P$. aeruginosa L6-1 and FA1 were similar to that of rhamnolipids produced from strain SG. The FTIR spectra of the anaerobically produced rhamnolipids from $P$. aeruginosa strains were also similar to that of reported rhamnolipids [30, 31]. All the FTIR spectra had the characteristic absorption bands of rhamnolipids. Absorption bands around $2927 \mathrm{~cm}^{-1}$,

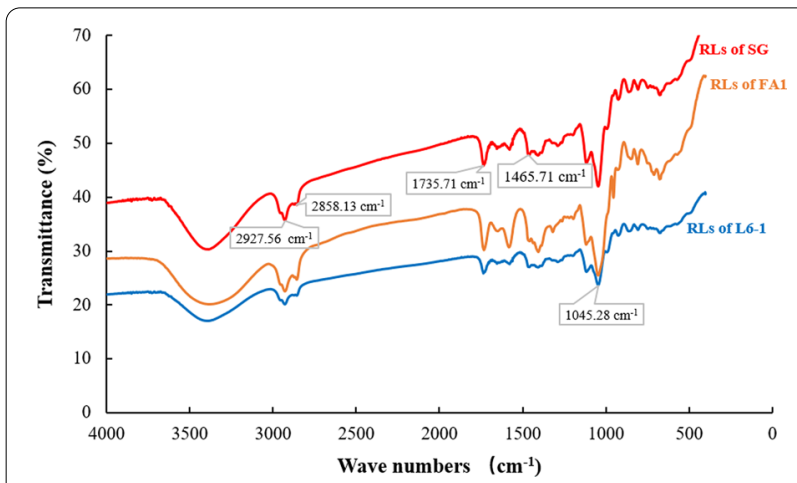

Fig. 5 Fourier Transform infrared (FTIR) spectra analysis of anaerobically produced rhamnolipids from $P$. aeruginosa strains using glycerol and nitrate. Anaerobically produced rhamnolipids by strain SG was used as the positive control
$2858 \mathrm{~cm}^{-1}$ and $1465 \mathrm{~cm}^{-1}$ were caused by the $\mathrm{C}-\mathrm{H}$ stretching vibrations of aliphatic groups. The absorption bands around $1735 \mathrm{~cm}^{-1}$ was caused by the ester groups. All the FTIR spectra also had the absorption area between $1452 \mathrm{~cm}^{-1}$ and $1045 \mathrm{~cm}^{-1}$ causing by the $\mathrm{C}-\mathrm{H}$ and $\mathrm{O}-\mathrm{H}$ vibrations. These are typical vibrations for carbohydrates. Results showed that $P$. aeruginosa strains did anaerobically produce rhamnolipids when using glycerol as carbon source.

\section{Activity of anaerobically produced rhamnolipids using glycerol}

The rhamnolipids-water solutions $(200 \mathrm{mg} / \mathrm{l})$ were prepared using the extracted rhamnolipids products. All the anaerobically produced rhamnolipids using glycerol decreased the air-water surface tension from 72.6 $\mathrm{mN} / \mathrm{m}$ to lower than $29 \mathrm{mN} / \mathrm{m}$. Studies reported the aerobically produced rhamnolipids can decrease the airwater surface tension to lower than $27 \mathrm{mN} / \mathrm{m}$ [32]. The anaerobically produced rhamnolipids from $P$. aeruginosa exhibited excellent surface activity as well. Good surface activity helps to change contact angle, increase wetting activity and even facilitate wetting reversal. The excellent surface activity can also improve the capillary effect and facilitate the flow of groundwater or oil in porous media. These are of great significance for enhanced oil recovery and pollution remediation [33]. Anaerobically produced rhamnolipids using glycerol also showed better emulsifying activity to crude oil with $\mathrm{EI}_{24}$ values higher than $65 \%$. Studies reported the aerobically produced rhamnolipids can emulsified crude oil with $\mathrm{EI}_{24}$ values ranging from 53 to $90 \%[32,34]$. The anaerobically produced rhamnolipids from $P$. aeruginosa also exhibited good emulsifying activity, which would be significant for enhanced oil recovery and pollution remediation $[4,35,36]$. The good emulsifying activity of biosurfactants can assist oil dispersion in oil reservoir and reduce oil viscosity [37]. Besides, the excellent emulsification effect can increase the solubility and the bioavailability of hydrophobic pollutants [36, 38].

\section{Glycerol intermediates and crude glycerol for anaerobic production of rhamnolipids}

Glycerol is water soluble and easy to be absorbed by microorganisms. In the present study, only glycerol support $P$. aeruginos $a$ to anaerobically produce rhamnolipids. Did the possible glycerol intermediates, hydroxyacetone, 1, 2-propylene glycol and 1, 3-propylene glycol, support for the anaerobic production of rhamnolipids by $P$. aeruginosa? As shown in Fig. 6, P. aeruginosa strains can anaerobically grow well using the possible glycerol intermediates. These substrates are involved in the glycolysis and gluconeogenesis pathways, which may be conducive to be rapidly absorbed and anaerobically metabolized by 


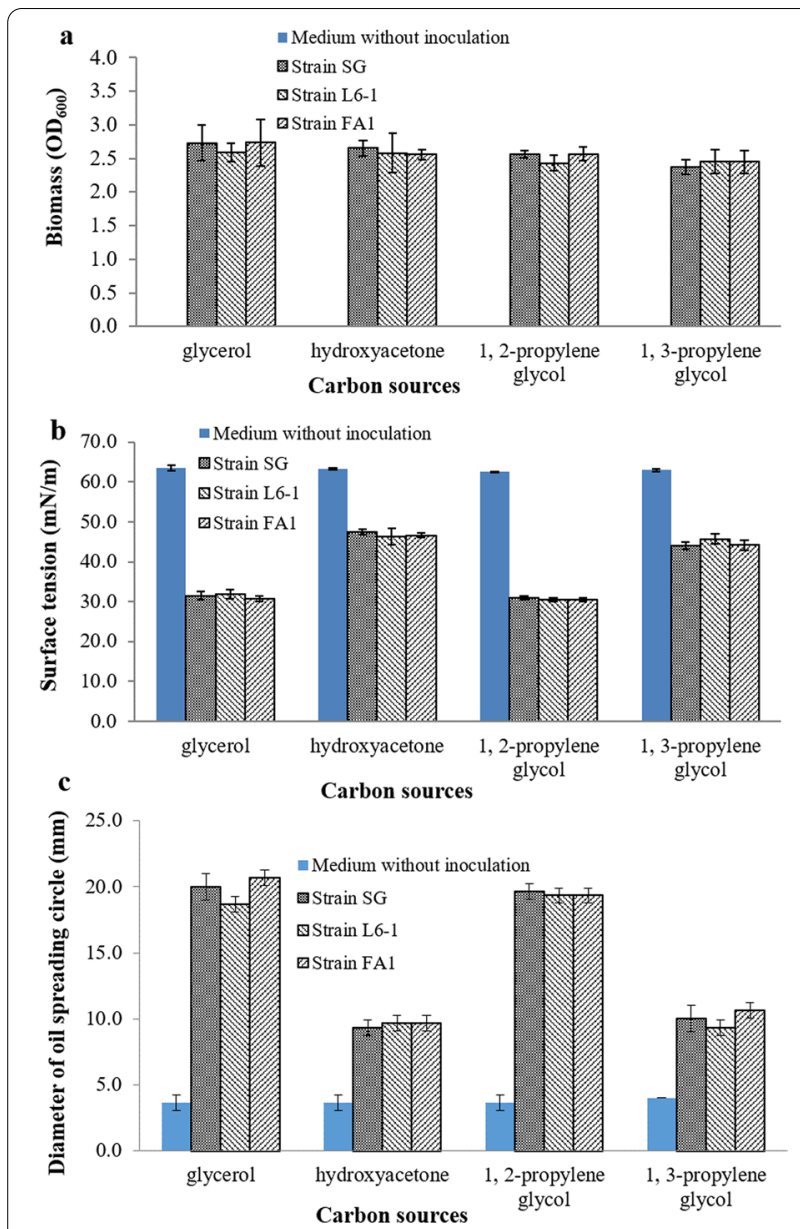

Fig. 6 Anaerobic production of rhamnolipids by P. aeruginosa strains using crude glycerol

P. aeruginosa [39]. P. aeruginosa strains obtained the biomass with $\mathrm{OD}_{600}$ values between 2.00 and 3.00 (Fig. 6a). Merely 1, 2-propylene glycol supported $P$. aeruginosa strains to anaerobically produce rhamnolipids, reducing the surface tension of anaerobic culture to about $31 \mathrm{mN} / \mathrm{m}$ (Fig. 6b). Using 1, 2-propylene glycol, the oil spreading circles diameters formed by anaerobic culture were about $20 \mathrm{~mm}$ (Fig. 6c).

Results demonstrated that 1, 2-propylene glycol, similar to glycerol, can make $P$. aeruginosa to anaerobically biosynthesize rhamnolipids. Hauser and Karnovsky cultured $P$. aeruginosa strain using ${ }^{14} \mathrm{C}$-labeled glycerol$\alpha-{ }^{14} \mathrm{C}$ and glycerol- $\beta-{ }^{14} \mathrm{C}$ under aerobic conditions. And they found that the $\mathrm{C}_{6}$ unit of rhamnose group in rhamnolipids product was directly condensed from the two molecules glycerol $\left(C_{3}\right.$ unit) without carbon chain rearrangement [40]. The polyol can be oxidized into dihydroxyacetone (DHA). DHA can be converted into dihydroxyacetone phosphate (DHAP) which is involved

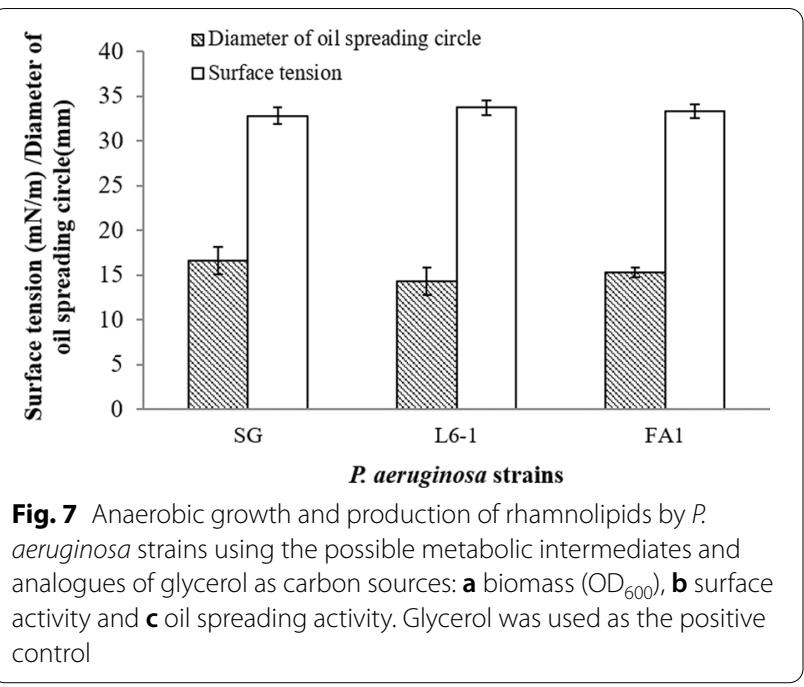

in glycolysis and gluconeogenesis pathways [39, 41]. Then glycolysis and gluconeogenesis pathways are connected with the de novo synthesis of fatty acids. Therefore, $P$. aeruginosa can metabolize glycerol and 1,2-propylene glycol to biosynthesize both the two precursors of rhamnolipids, TDP-L-rhamnose and $\beta$-hydroxy fatty acids. Glycerol metabolism facilitate the anaerobic biosynthesis of rhamnolipids in P. aeruginosa.

As shown in Fig. 7, three $P$. aeruginosa strains anaerobically produced rhamnolipids using crude glycerol. The surface tension of anaerobic culture was decreased to below $35 \mathrm{mN} / \mathrm{m}$. The oil spreading circles diameters formed by anaerobic culture using crude glycerol were about $15 \mathrm{~mm}$. Results showed that crude glycerol can be used for anaerobic production of rhamnolipids by $P$. aeruginosa strains as well. Previous studies reported aerobic production of rhamnolipids by $P$. aeruginosa using crude glycerol as low-cost substrate [42, 43]. In the present study, three $P$. aeruginosa strains achieved producing rhamnolipids anaerobically when using crude glycerol as substrates.

\section{Prospects and bottlenecks to anaerobic production of rhamnolipids}

Although $P$. aeruginosa and its rhamnolipids have been extensively studied, studies on anaerobic production of rhamnolipids by $P$. aeruginosa are relatively less. Only a few studies reported that some rare $P$. aeruginosa strains can produce rhamnolipids anaerobically. The decisive factors for anaerobic production of rhamnolipids were unknown. Two possible hypotheses on the decisive factors for anaerobic production of rhamnolipids were proposed, the strains specificity of rare 
P. aeruginosa (hypothesis 1 ) and the effect of specific substrates (hypothesis 2). This study answered hypothesis 2 is credible. Glycerol substrate rather than the strain specificity contributed to the anaerobic production of rhamnolipids by $P$. aeruginosa. $P$. aeruginosa strains achieved producing rhamnolipids anaerobically when using glycerol or crude glycerol as substrates. Strain specificity resulted in the different anaerobic yield of rhamnolipids. Results are helpful to advance the research on anaerobic production of rhamnolipids, deepen the rhamnolipids biosynthesis theory and guide the optimization process for anaerobic production of rhamnolipids.

Anaerobic production of rhamnolipids can meet the in situ applications in anoxic environments, such as oil reservoirs, deep soil, sediments [7-9]. Based on anaerobic production of rhamnolipids, fermentation without aeration can avoid the foam problem in rhamnolipids production $[14,15]$. The anaerobic production of rhamnolipids has important research and application significance.

But the anaerobic yield of rhamnolipids from $P$. aeruginosa using glycerol were lower than the aerobic yield of rhamnolipids [9]. The low anaerobic yield of rhamnolipids would limit its applications. Enhancing the anaerobic production of rhamnolipids is in demand. The anaerobic biosynthesis mechanism of rhamnolipids is significant for metabolic engineering of $P$. aeruginosa to enhance anaerobic yield. The biosynthesis pathways and key genes involved in anaerobic production of rhamnoipids have been revealed [44]. The anaerobic yield of rhamnolipids by $P$. aeruginosa can be enhanced by medium optimization, pathways regulation and genes modification.

Compared with anaerobic conditions, aerobic conditions are conducive to biosynthesize reducing power $\left(\mathrm{NADPH}\right.$ or $\left.\mathrm{NADH}_{2}\right)$, which promotes fatty acid synthesis and ultimately enhances rhamnolipids synthesis. Increasing the reducing power (NADPH or $\mathrm{NADH}_{2}$ ) level will also probably enhance the anaerobic production of rhamnolipids by $P$. aeruginosa. $\beta$-hydroxy fatty acids are one of two key precursors in rhamnolipids biosynthesis. $\beta$-hydroxy fatty acids are derived from the de novo synthesis of fatty acids and $\beta$-oxidation of fatty acids [45]. Fatty acids metabolism requires a lot of reducing power. The reducing power maybe a limiting factor for anaerobic metabolism of fatty acids. In this study, glycerol is a more reductive carbon source than other tested glucose. Using glycerol as carbon source is conducive to reducing power synthesis for $P$. aeruginosa, which promotes fatty acids metabolism and rhamnolipids production under anaerobic conditions.

\section{Conclusions}

In this study, two possible hypotheses on the decisive factors for anaerobic production of rhamnolipids were proposed, the strains specificity of rare $P$. aeruginosa (hypothesis 1) and the effect of specific substrates (hypothesis 2). Glycerol supported all tested $P$. aeruginosa strains to produce rhamnolipids anaerobically. Hypothesis 2 is credible. Glycerol substrate rather than the strain specificity contributed to the anaerobic production of rhamnolipids by $P$. aeruginosa. Strain specificity resulted in the different anaerobic yield of rhamnolipids. Nitrate was the excellent nitrogen source for anaerobic production of rhamnolipids. FTIR spectra analysis confirmed the anaerobically produced rhamnolipids by three $P$. aeruginosa strains using glycerol. Anaerobically produced rhamnolipids exhibited good surface activity and emulsifying activity. P. aeruginosa also produced rhamnolipids anaerobically using 1 , 2-propylene glycol and crude glycerol. Prospects and bottlenecks to anaerobic production of rhamnolipids were also discussed. Results are helpful to advance the research on anaerobic production of rhamnolipids.

\section{Acknowledgements \\ This work was mainly supported by the National Natural Science Foundation of China (31700117) and the Introduced Talent Research Start-up Fund of Qufu Normal University (6096).}

\section{Authors' contributions}

FZ conceived and designed the study, carried out the experiments and drafted the manuscript. YTW participated in analyzing the experimental data. QZW participated in biosurfactants extraction and FTIR analysis. MYZ participated in bacterial cultivation and determination. QFC assisted in revising the manuscript. All authors read and approved the final manuscript.

\section{Funding}

This work was financially supported by the National Natural Science Foundation of China (31700117), the Introduced Talent Research Start-up Fund of Qufu Normal University (6096), and the Young Talents Invitation Program of Shandong Provincial Colleges and Universities.

\section{Availability of data and materials}

The datasets supporting the conclusions of this article are included within the article.

\section{Declarations}

Ethics approval and consent to participate Not applicable.

\section{Consent for publication}

Not applicable.

\section{Competing interests}

The authors declare that they have no competing interests.

\section{Author details}

${ }^{1}$ School of Life Sciences, Qufu Normal University, Qufu 273165, Shandong, China. ${ }^{2}$ Research Institute of Petroleum Exploration and Development (Langfang), Langfang 065007, Hebei, China.

Received: 6 July 2021 Accepted: 12 September 2021

Published online: 23 September 2021 


\section{References}

1. Müller MM, Kügler JH, Henkel M, Gerlitzki M, Hörmann B, Pöhnlein M, Syldatk C, Hausmann R. Rhamnolipids-next generation surfactants? J Biotechnol. 2012;162:366-80

2. Varjani S, Upasani VN. Critical review on biosurfactant analysis, purification and characterization using rhamnolipid as a model biosurfactant. Bioresour Technol. 2017;232:389-97.

3. Chong H, Li Q. Microbial production of rhamnolipids: opportunities, challenges and strategies. Microb Cell Fact. 2017;16(1):137.

4. Gudiña EJ, Rodrigues Al, Alves E, Domingues MR, Teixeira JA, Rodrigues LR. Bioconversion of agro-industrial by-products in rhamnolipids toward applications in enhanced oil recovery and bioremediation. Bioresour Technol. 2015;177:87-93.

5. Cameotra SS, Singh P. Synthesis of rhamnolipid biosurfactant and mode of hexadecane uptake by Pseudomonas species. Microb Cell Fact. 2009;8(1):16.

6. Zou H, Du W, Ji M, Zhu R. Enhanced electrokinetic remediation of pyrene-contaminated soil through $\mathrm{pH}$ control and rhamnolipids addition. Environ Eng Sci. 2016;33(7):507-13.

7. Domingues PM, Almeida A, Leal LS, et al. Bacterial production of biosurfactants under microaerobic and anaerobic conditions. Rev Environ Sci Biotechnol. 2017;16(2):239-72.

8. Zhao F, Zhang J, Shi R, Han S, Ma F, Zhang Y. Production of biosurfactant by a Pseudomonas aeruginosa isolate and its applicability to in situ microbial enhanced oil recovery under anoxic conditions. RSC Adv. 2015;5(45):36044-50

9. Zhao F, Shi R, Ma F, et al. Oxygen effects on rhamnolipids production by Pseudomonas aeruginosa. Microb Cell Fact. 2018;17:39.

10. Câmara J, Sousa M, Neto ELB, et al. Application of rhamnolipid biosurfactant produced by Pseudomonas aeruginosa in microbial-enhanced oil recovery (MEOR). J Pet Explor Prod Technol. 2019;9(3):2333-41.

11. Youssef NH, Simpson DR, Mclnerney MJ, et al. In-situ lipopeptide biosurfactant production by Bacillus strains correlates with improved oil recovery in two oil wells approaching their economic limit of production. Int Biodeterior Biodegrad. 2013;81:127-32.

12. Zhao F, Li P, Guo C, Shi R, Zhang Y. Bioaugmentation of oil reservoir indigenous Pseudomonas aeruginosa to enhance oil recovery through in-situ biosurfactant production without air injection. Bioresour Technol. 2018;251:295-302.

13. Albino JD, Namb IM. Partial characterization of biosurfactant produced under anaerobic conditions by Pseudomonas sp ANBIOSURF-1. Adv Mater Res. 2010;93:623-6.

14. Anic I, Apolonia I, Franco P, et al. Production of rhamnolipids by integrated foam adsorption in a bioreactor system. AMB Expr. 2018;8:122.

15. Long X, Shen C, He N, et al. Enhanced rhamnolipids production via efficient foam-control using stop valve as a foam breaker. Bioresour Technol. 2017;224:536-43.

16. Arai $\mathrm{H}$. Regulation and function of versatile aerobic and anoxic respiratory metabolism in Pseudomonas aeruginosa. Front Microbiol. 2011;2:1-13.

17. Schobert M, Jahn D. Anaerobic physiology of Pseudomonas aeruginosa in the cystic fibrosis lung. Int J Med Microbiol. 2010;300:549-56.

18. Chayabutra C, Wu J, Ju LK. Rhamnolipids production by Pseudomonas aeruginosa under denitrification: effects of limiting nutrients and carbon substrates. Biotechnol Bioeng. 2001;72:25-33.

19. Shekhar S, Sundaramanickam A, Balasubramanian T. Biosurfactant producing microbes and their potential applications: a review. Crit Rev Environ Sci Technol. 2015;45(14):1522-54.

20. Zhao F, Shi R, Zhao J, et al. Heterologous production of Pseudomonas aeruginosa rhamnolipid under anaerobic conditions for microbial enhanced oil recovery. J Appl Microbiol. 2015;118(2):379-89.

21. Zhao F, Zhou J, Han S, Ma F, Zhang Y, Zhang J. Medium factors on anaerobic production of rhamnolipids by Pseudomonas aeruginosa SG and a simplifying medium for in situ microbial enhanced oil recovery applications. World J Microbiol Biotechnol. 2016;32(4):54.

22. Zhao F, Liang X, Ban Y, Han S, Zhang J, Zhang Y, Ma F. Comparison of methods to quantify rhamnolipids and optimization of oil spreading method. Tenside Surfact Det. 2016;53(3):243-8.

23. Nicolò MS, Cambria MG, Impallomeni G, Rizzo MG, Pellicorio C, Ballistreri A, Guglielmino SP. Carbon source effects on the mono/dirhamnolipid ratio produced by Pseudomonas aeruginosa L05, a new human respiratory isolate. New Biotechnol. 2017;39:36-41.
24. Ehinmitola EO, Aransiola EF, Adeagbo OP, et al. Comparative study of various carbon sources on rhamnolipid production. S Afr J Chem Eng. 2018;26:42-8.

25. Tan YN, Li Q. Microbial production of rhamnolipids using sugars as carbon sources. Microb Cell Fact. 2018;17(1):1-13.

26. Kerr ED, Schulz BL. Vegemite Beer: yeast extract spreads as nutrient supplements to promote fermentation. PeerJ. 2016;4:e2271.

27. Reis RS, Pereira AG, Neves BC, Freire DMG. Gene regulation of rhamnolipid production in Pseudomonas aeruginosa-a review. Bioresour Technol. 2011;102:6377-84.

28. Soberón-Chávez G, Lepine F, Déziel E. Production of rhamnolipids by Pseudomonas aeruginosa. Appl Microbiol Biotechnol. 2005;68:718-25.

29. Reis RS, Rocha SLG, Chapeaurouge DA, Domont GB, Santa Anna LMM, Freire DMG, Perales J. Effects of carbon and nitrogen sources on the proteome of Pseudomonas aeruginosa PA1 during rhamnolipid production. Process Biochem. 2010;45:1504-10.

30. Leitermann F, Syldatk C, Hausmann R. Fast quantitative determination of microbial rhamnolipids from cultivation broths by ATR-FTIR Spectroscopy. J Biol Eng. 2008;2:13-20.

31. Kiefer J, Radzuan MN, Winterburn J, et al. Infrared spectroscopy for studying structure and aging effects in rhamnolipid biosurfactants. Appl Sci. 2017;7(5):533.

32. Nitschke M, Cost SGVAO, Contiero J. Rhamnolipid surfactants: an update on the general aspects of these remarkable biomolecules. Biotechnol Prog. 2005;21(6):1593-600.

33. Park T, Jeon M, Yoon S, et al. Modification of interfacial tension and wettability in Oil-Brine-Quartz system by in situ bacterial biosurfactant production at reservoir conditions: implications for microbial enhanced oil recovery. Energy Fuels. 2019;33(6):4909-20.

34. Pekdemir T, Copur M, Urum K. Emulsification of crude oil-water systems using biosurfactants. Process Saf Environ. 2005;83(1):38-46.

35. Rahman KSM, Rahman TJ, Kourkoutas Y, Petsas I, Marchant R, Banat IM. Enhanced bioremediation of n-alkane in petroleum sludge using bacterial consortium amended with rhamnolipids and micronutrients. Bioresour Technol. 2003;90(2):159-68.

36. Varjani SJ, Upasani VN. Core Flood study for enhanced oil recovery through ex-situ bioaugmentation with thermo-and halo-tolerant rhamnolipids produced by Pseudomonas aeruginosa NCIM 5514. Bioresour Technol. 2016;220:175-82.

37. Hosseininoosheri P, Lashgari HR, Sepehrnoori K. A novel method to model and characterize in-situ bio-surfactant production in microbial enhanced oil recovery. Fuel. 2016;183:501-11.

38. Sponza DT, Gök O. Effect of rhamnolipids on the aerobic removal of polyaromatic hydrocarbons (PAHs) and COD components from petrochemical wastewater. Bioresour Technol. 2010;101(3):914-24.

39. Pobletecastro I, Wittmann C, Nikel PI. Biochemistry, genetics and biotechnology of glycerol utilization in Pseudomonas species. Microb Biotechnol. 2020;13(1):32-53.

40. Hauser G, Karnovsky ML. Rhamnose and rhamnolipide biosynthesis by Pseudomonas aeruginosa. J Biol Chem. 1957;224(1):91-105.

41. Biebl H, Menzel K, Zeng AP, Deckwer WD. Microbial production of 1,3-propanediol. Appl Microbiol Biotechnol. 1999;52:289-97.

42. Eraqi WA, Yassin AS, Ali AE, et al. Utilization of crude glycerol as a substrate for the production of rhamnolipid by Pseudomonas aeruginosa. Biotechnol Res Int. 2016;2016:3464509.

43. Zhao $F$, Jiang $H$, Sun $H$, et al. Production of rhamnolipids with different proportions of mono-rhamnolipids using crude glycerol and a comparison of their application potential for oil recovery from oily sludge. RSC Adv. 2019;9(6):2885-91.

44. Zhao F, Wang Q, Zhang Y, et al. Anaerobic biosynthesis of rhamnolipids by Pseudomonas aeruginosa: performance, mechanism and its application potential for enhanced oil recovery. Microb Cell Fact. 2021;20:103.

45. Abdel-Mawgoud AM, Lépine F, Déziel E. A stereospecific pathway diverts $\beta$-oxidation intermediates to the biosynthesis of rhamnolipid biosurfactants. Chem Biol. 2014;21(1):156-64.

\section{Publisher's Note}

Springer Nature remains neutral with regard to jurisdictional claims in published maps and institutional affiliations. 